Review

\title{
Thinking outside the Box: Could there be a Plant-Based Treatment for Coronavirus Infections? A Review of the Literature
}

Sharon Clevenger ${ }^{1,2,3, *}$

1. Huntington College of Health Sciences, Knoxville, TN, USA; E-Mail: sfclevenger@iccbt.org

2. Indiana Center for Cognitive Behavior Therapy, Fort Wayne, IN, USA

3. Department of Psychology, Purdue University, IN, USA

* Correspondence: Sharon Clevenger; E-Mail: sfclevenger@iccbt.org

Academic Editor: Gerhard Litscher

OBM Integrative and Complementary Medicine

2020, volume 5, issue 2

doi:10.21926/obm.icm.2002031
Received: April 20, 2020

Accepted: May 10, 2020

Published: May 18, 2020

\section{Abstract}

The novel coronavirus COVID-19 is a viral pathogen that first emerged in Wuhan, China in 2002 and has spread to 202 countries (as of March 28, 2020) [1]. Despite the fact that the 2005 SARSCoV pandemic infected over 8,500 people, and caused over 800 deaths with billions of dollars in economic loss worldwide, as of 2015 there were still no clinically approved antiviral drugs, vaccines or monoclonal antibody therapies to treat SARS-CoV infections [2]. Antiviral medications that have been used to treat or control other viral infections have been ineffective and therefore, it is imperative to consider alternatives to current antiviral treatment options for this disease. Essential oils (EO) and their isolated compounds, (terpenes, terpenoids and aromatic compounds) have been shown to have antimicrobial activity against a wide range of pathogens including bacteria, viruses and fungi [3]. The mechanism of action of EO's is linked to 100 's of chemical compounds per plant extract, and the fact that they are lipophilic, easily passing through viral cell walls. The presence of nanoparticles (NPs) functionalized within the EO render the EO impervious to the defenses of pathogens. The permeability eventually results

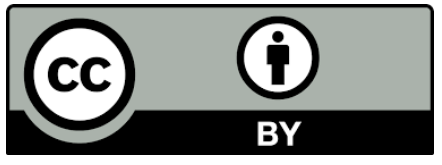

(C) 2020 by the author. This is an open access article distributed under the conditions of the Creative Commons by Attribution License, which permits unrestricted use, distribution, and reproduction in any medium or format, provided the original work is correctly cited. 
in the death of pathogenic cell as leakage of critical molecules and ions occurs destroying the cell [4].

\section{Introduction and Background}

The novel coronavirus COVID-19 (coronavirus disease 2019) is a viral pathogen that first emerged in humans in Wuhan, China in 2002 and has now spread to 202 countries (as of March 28, 2020) [1]. COVID-19 causes severe acute respiratory coronavirus (SARS-CoV), a disease that has caused a worldwide epidemic with severe morbidity and high mortality rates, particularly in older individuals. COVID-19 is a by-product of the rapid economic growth in southern China leading to increased demand for animal proteins, including those from exotic game food animals such as civets and pangolins. A civet is a meat-eating mammal with a somewhat cat-like appearance with a small head, long body, and long tail. Its muzzle is long and often pointed, rather like that of an otter or a mongoose. Excluding its tail, a civet ranges from about 17 inches to 28 inches long, and weighs between three to 10 pounds. There are several species of pangolins, an endangered species that are best described as scaly ant-eating mammals that range in size from 8-73 pounds [5]. Civets and pangolins are poached for their meat and are often found in large numbers in overcrowded cages with little to no biosecurity measures in "wet-markets". The wet-markets are thought to be the cause that allowed this novel virus to "jump" from animals to humans [6]. Chinese wet-markets are unique epicenters for transmission of potential viral pathogens in close proximity to humans, and have extremely high viral burden or strains of higher transmission efficiency facilitating the transmission of viruses to humans [7]. In 2007, researchers identified that the small reemergence of SARS in late 2003 after the resumption of the wildlife wet-market in southern China resulted in the discovery of a very similar novel virus in horseshoe bats. The discovery of bat SARS-CoV suggested that SARS could return if conditions remained fit for the introduction, mutation, amplification, and transmission of this dangerous virus [7]. Despite the fact that the 2005 SARS-CoV pandemic infected over 8,500 people, and caused over 800 deaths with billions of dollars in economic loss worldwide, as of 2015 there were still no clinically approved antiviral drugs, vaccines or monoclonal antibody therapies to treat SARSCoV infections [2].

\section{Overview of Viral Pathophysiology}

Viruses are exceedingly small (about 20 - 200 nanometers in diameter) and usually round, consisting of a tiny piece of genetic material surrounded by a thin protein coating (capsid) or a thin, fatty envelope. Human viruses have extremely small genomes consisting of either doublestranded DNA that replicate inside the host cell nucleus, or single-stranded RNA viruses, like SARS$\mathrm{CoV}$, that replicate in the host cell cytosol [8]. Viruses can be differentiated from other pathogens in that they are the only group of microorganisms that cannot replicate outside of a host cell. In addition, viruses as compared to bacteria, do not consume cellular nutrients directly, instead they 
hijack the hosts cellular machinery for their own energy purposes [9]. There are essentially three possible steps that a viral pathogen may take to overcome host immune system defenses when creating an infection: 1) subvert or defeat the hosts innate defense system, 2) create permissive "niche" for replication, and 3) develop antigenic variation to escape detection by host defenses [9]. Once the virus gains control of the hosts cellular machinery, the virus injects its own genetic material into the cytosol with all of the information needed to replicate itself. It does this by taking control of the cells genetic replicating machinery and forces the host cell to replicate the virus with its nutrients and energy sources thereby preventing the host cell from conducting normal processes for survival. The result of hijacking the host cells is that it usually kills the host cell. When the host cell dies, the virus is released along with new viruses that go on to repeat the process of infecting new cells [10].

There are basically four types of viral infections, classified clinically: 1) Transient acute, 2) Chronic latent, 3) Chronic, 4) and Transforming. The Epstein Barr Virus (EBV) for example, is a double-stranded DNA transforming virus, and like most viruses, the genome encodes for three types of proteins. One protein is intended for replication of the genome, another for packaging the genome for delivery to more cells, and finally, a protein for modifying the structure and function of the host cell to enhance its own replication [8]. The steps of viral infection and replication include: 1) entry into the host cell, 2) disassembly of the infectious virus particle, 3) replication of the viral genome, 4) transcription of viral genes and synthesis of viral proteins, 5) assembly of these viral components into progeny virus particles, and finally 6) release of progeny virions to go on to infect other cells [8].

Viruses are encapsulated with a protein coat called a capsid. A capsid packaged with the viral genome is called a nucleocapsid; which, can be enveloped with a fatty outer layer, or nonenveloped. Nucleocapsids of nonenveloped viruses usually leave an infected cell by lysing it which kills the host cell. Enveloped viruses have their nucleocapsid enclosed within a lipid bilayer membrane that the virus acquires from the host cell in the process of budding off from the hostcell plasma membrane. An enveloped nucleocapsid can bud and leave the host cell without disrupting the membrane, may kill the cell by taking all of its nutrients for itself, or it might leave the cell intact and hide causing persistent infections that may last for years, often without noticeable deleterious effects on the host [8]. The SARS-CoV virus is an RNA virus that kills the cell and then releases its viral particles to go on to infect other cells in a cyclical process of infectionrelease-reinfection. In the case of SARS-CoV, the most common tissues attacked are the alveolar lung tissues. The infectious process causes decreased normal lung function such as the ability to remove mucous and debris, destruction of the alveolar cells lining the lungs thereby preventing oxygen from being absorbed and carbon dioxide from being exhaled [11]. Essentially, lung function ceases, pneumonia develops, and the person suffocates to death.

\section{Corona-Type RNA Viruses}

Most influenza viruses and coronaviruses are enveloped RNA viruses which means that they are covered by a membrane ( $M$ protein) of cellular matter taken from their host cell. RNA viruses are 
unable to replicate on their own because unlike DNA, RNA does not contain self-correcting mechanisms. RNA is the active, reproducing template copied from strands of DNA. In cases of RNA viruses like SARS-CoV, the viral genetic code is organized as single-stranded RNA and is the largest of the known RNA genomes. RNA viruses replicate rapidly, mutate often and are emerging rapidly on the world stage. Some of the emerging RNA-virus related diseases include Ebolavirus, Middle East respiratory syndrome (MERS), SARS-CoV, Influenza A virus (H1N1, IAV) Zika virus, Human Immunosuppressive Virus (HIV) and West Nile Virus [5]. For an enveloped virus to invade host cells, the viral envelope must be removed for the inner part of the virus particle to enter the cytoplasm of the host cell, where it can then reproduce. A nucleocapsid (N) protein complex with the genome RNA forms a helical capsid structure that is found within the viral envelope. Trimers of the spike proteins form the peplomers embedded in the lipid envelope that radiate outward giving the virion its "corona" or "crown-like" appearance under an electron microscope [11]. Coronaviruses and influenza viruses infect a host cell by fusing their viral envelope to the outer layer of the host cell. The host cell membrane is then dissolved or punctured to increase the connection with the viral envelope. Once the viral envelope is firmly attached to the host cell, the virus can enter the cell, essentially by the host cell engulfing it the way it would any food particle [11]. The main differences between COVID-19 and seasonal influenza is that death rate from seasonal flu is typically around $0.1 \%$ in the U.S., but the death rate of COVID-19 is ranging between 1-3\% with elderly and immuno-compromised individuals hardest hit. COVID-19 also has a longer incubation period (10-14 days compared to 2-3 days) where the person is not sick but is shedding the virus. In addition, there are antiviral medications and vaccines available for influenza and neither is available yet for COVID-19 [12].

Similar to other coronaviruses, SARS-CoV is thought to attach by the hydrophobic domains of their replication machinery to the limiting membrane of autophagosomes forming double-membrane vesicles. Once sufficient viral genomic RNA and structural proteins are accumulated, viral assembly by budding of the helical nucleocapsid at the endoplasmic reticulum to the Golgi intermediate compartment occurs. The coronavirus membrane $(\mathrm{M})$ protein is the critical component in virion assembly as one its primary functions is to mediate the incorporation of the spikes (S proteins) into the viral envelope. RNA Viral M proteins are integrated into the lipid bilayer in and, in general, can be simple, spanning the membrane only once; or complex, traversing the membrane multiple times. The coronaviruses have triple-membrane-spanning $M$ proteins that interact with the nucleocapsid (N) proteins and viral RNA to generate the basic viral structure, and to interact with the envelope and spike proteins to induce viral budding and release. Unlike other coronaviruses, what makes SARS-CoV $M$ proteins unique is the incorporation of another triple-membrane-spanning protein: the SARS-CoV open reading frame-3a (ORF3a) protein which is an accessory protein into the virion [6, 7]. The SARSCoV ORF3a accessory protein activates the nod-like receptor family, pyrin domain-containing-3 (NLRP3) protein that regulates the secretion of proinflammatory cytokines interleukin-1-beta (IL-1 $\beta$ ) and IL-18. Activation of the NLRP3 inflammasome also promotes tumor necrosis factor (TNF) receptor-associated factor-3 (TRAF3)-mediated ubiquitination of apoptosis-associated speck-like protein containing a caspase recruitment domain [13]. 


\section{Pathogenesis of SARS-CoV}

Patients infected with SARS-CoV most frequently initially presented with fever, nonproductive cough, sore throat, headache and myalgia, with dyspnea often not becoming a prominent feature until days 7-14 of the illness. During the second phase of the illness, dyspnea and hypoxia, often with continued fever and frequently accompanied by diarrhea, became more prominent. As the respiratory status continues to deteriorate, acute respiratory distress syndrome (ARDS) develops often requiring mechanical respiration by the third week [11]. Similar to other causes of atypical pneumonia, physical signs upon chest examination are minimal compared with the radiographical (Xray) findings [1]. SARS-CoV causes damage to the airway epithelial cells, which means that the lungs are unable to clear the debris or mucus frequently resulting in pneumonia. There is also evidence of a "cytokine storm", which is the dramatic development of pulmonary destruction caused by rapid increases in levels of chemokine and cytokine proinflammatory molecules, often complicated further by pneumonia. Chest radiographs typically show "ground-glass opacities" and focal consolidations, especially in the periphery and subpleural regions of the lower zones. Progressive involvement of both lungs is not uncommon [6]. Deaths have occurred as early as day 4 and as late as 108 days after onset of symptoms. Virus shedding from the respiratory tract generally peaks around day 10 and subsequently declines. Virus excretion from the GI tract is also frequently present with IgG antibodies detected 10-15 days after onset of symptoms. The severity of the disease is correlated with increasing age, with mortality reaching $50 \%$ for patients over $60[7,11]$.

\section{Treatment of Viral Disease}

Eradication of diseases is based on a principle of threshold proportion: if a certain proportion of a population is immune to a disease, the pathogen that causes that disease will be unable to reproduce at a high enough level to maintain the pathogen population. That is because over time, the infected hosts will either recover, or die, leaving too few susceptible hosts for the pathogen to infect halting further spread. Sometimes this phenomenon is called "herd immunity" [14]. Hepatitis C (Hep C) for example, is an RNA virus that has wide variability in both progression and presentation of symptoms due to high variability in RNA genotypes. Hep C may be very slow to progress with little to no symptoms in the acute phase; however, it will progress to a chronic state in $85-90 \%$ of individuals who contract the disease [15]. Mutations of the RNA virus cause it to avoid the body's immune system responses allowing it to live and replicate undetected in the liver causing inflammation, fibrosis, and scar tissue development. The most severe outcome is death of liver tissues to the point of cirrhosis of the liver, and possibly liver cancer [15]. Success of conventional treatment with interferon and ribavirin depends on the genotype of the specific virus, and the extent to which the viral load has expanded.

Searches for effective medications to treat or prevent SARS-CoV has led to studies looking at drugs currently on the market to see if they may potentially work against the virus. One such study looked at disulfiram, an aldehyde dehydrogenase inhibitor used as an anti-alcohol use medication. The researchers found that disulfiram acted as an allosteric inhibitor of Middle East respiratory syndrome 
coronavirus (MERS-CoV) papain-like protease (PLpro), and as a competitive (or mixed) inhibitor of SARS-CoV PLpro. Unfortunately, disulfiram has a number of side-effects that may make it unpopular in vivo [16]. Options for biologic agents that are well-tolerated and effective has led to research into potential plant-based treatments. Phytochemicals in essential oils (EO) for example, have been shown to have complementary and overlapping mechanisms of action; which, result in their antiviral effects through inhibition of the formation of viral DNA or RNA, or directly inhibiting the activity of viral reproduction [17].

A study conducted by Giraud-Robert (2005) evaluating the antiviral potential for treatment of hepatitis-C (HCV) found that EO's may be effective as a monotherapy, or in conjunction with treatment as usual (TAU) [18]. The study enrolled 60 individuals who were chronic carriers of hepatitis B (HBV) or C (HCV) (50 HCV and $10 \mathrm{HBV})$. The EO's chosen for evaluation included Ravensara, Rhododendron groenlandicum (Labrador tea), carrot seed, Thymus vulgaris thujanoliferum (thujanol), Laurus nobilis (bay laurel), Melaleuca quinquenervia (niaouli) and helichrysum were used orally, either alone or together with TAU. In patients with HCV that were treated with bi-therapy plus EO's, tolerance and response to treatment was improved (80\% good tolerance and 100\% remission especially for genotype-1). For those with HCV treated with EO's alone an improvement in hepatitis was noted in $64 \%$ of cases, and for those with HBV, two cures were obtained with EO's as monotherapy [18]. Essential oils as blends or as single oils have been reviewed extensively to evaluate their potential antimicrobial effects. One blend in particular reviewed by Brochot and Guilbot, et al (2017) that contained Eucalyptus globulus and Cinnamomum Zeylanicum EO was shown to have potent antiviral activity [3]. The main constituent ingredients (e.g. terpenes and phenylpropanoids), are thought to be responsible for their biological properties in a synergistic manner to act as antivirals [19]. Single EO's from E. globulus, Melaleuca alternifolia (tea tree) and thyme and their major monoterpene compounds alpha-terpinene, gamma-terpinene, alpha-pinene, p-cymene, terpinen-4-ol, alpha-terpineol, thymol, carvacrol, citral and 1,8-cineole were found effective against a specific virus: herpes simplex virus type-1 (HSV-1) in vitro by Astani, Reichling and Schnitzler P (2010) [19]. The monoterpene carvacrol is a phenol that is a natural monoterpene derivative of cymene. Used by itself, carvacrol is an inhibitor of bacterial growth used as a food additive and is a potent activator of the human ion channels transient receptor potential V3 (TRPV3) and A1 (TRPA1) [20]. Astani, Reichling and Schnitzler observed that single EOs from E. globulus and M. alternifolia reduced HSV-1 viral infectivity by $>96 \%$, the monoterpenes alone inhibited HSV by about $>80 \%$ [19]. Mixtures of different monoterpenes found in $M$. alternifolia EO showed a ten-fold higher selectivity index, and lower toxicity, than isolated single monoterpenes indicating that the chemical components act synergistically to exert antimicrobial/antiviral effects. Other individual EOs extracted from cinnamon tree bark that included isolated compounds, including terpenes and terpenoids and aromatic compounds such as the phenylpropanoid cinnamaldehyde and eugenol an allyl chain-substituted guaiacol, a member of the allylbenzene class of chemical compounds, have been shown to have antimicrobial activity against a wide range of pathogens including bacteria, viruses and fungi [3].

The proposed mechanism of action of EO's as effective antimicrobials is linked to their complex composition of 100's of chemical compounds acting synergistically per plant extract, and their cytotoxic effects that damages pathogenic cell membranes. The lipophilic properties of EOs account 
for their ability to pass easily through a viral, fungal, or bacterial cell wall and cytoplasmic membrane causing destruction of the structure of the polysaccharide, fatty acid, and phospholipid layers, making the membranes permeable to their effect inside the cell. The use of EO's as antimicrobials is not without risk. Since EOs do not specifically target pathogens; they also have the potential to affect eukaryotic cells in a reversible, or irreversible manner [3]. One component that contributes to the stability of EOs as antimicrobials is the presence of nanoparticles (NPs) functionalized within the EO itself. The NP's render the EO impervious to the defenses of pathogens due to their increased chemical stability and solubility, decreased rapid evaporation and minimized degradation of the active EO biochemical components. The application of encapsulated EO's also supports controlled and sustained release, enhancing bioavailability and efficacy against multidrug-resistant pathogens. EO's are also highly "hydrophobicity"; which is, the ability of EO's to permeate cellular lipid layers enabling them to partition themselves with the lipids present in the cell membrane of microbes and their mitochondria, disturbing the cells structure rendering the pathogenic microbe more permeable. The permeability eventually results in the death of pathogenic cell as leakage of critical molecules and ions occurs destroying the cell [4].

\section{Treatment of Coronaviruses}

According to the World Health Organization (WHO) website on coronavirus 2019, no pharmaceutical products have yet been shown to be safe and effective for the treatment of infections by COVID-19 [12]. The correlation between viral loads and clinical outcome suggests that suppression of viral replication by effective antiviral drugs should be the key to preventing morbidity and mortality; however, in vitro susceptibility test results are often conflicting, and disappointing. An alternative treatment to pharmaceutical antivirals may lie in herbal phytochemicals. Research on combination herbal formulations have been found to possess some antiviral activity against SARS-CoV in vitro [6]. The antimicrobial effects of extracts from plants are linked to their unique, complex composition and cytotoxic effects of the individual phytochemicals. Lipophilic characteristics allow EOs to pass through the cell membrane where they alter the structure of the polysaccharide, fatty acid, and phospholipid layers, making the viral membrane permeable $[3,21]$. Many essential oils (EO) exert their antiviral effects by either inhibiting the formation of viral DNA or RNA or, inhibiting the activity of viral reproduction (Figure 1) [17, 22]. Viruses in general are more difficult to eradicate due to having numerous invasion strategies with each strain having its own unique configuration of surface molecules. The surface molecules act like a key in a lock mechanism enabling the viruses to enter into hosts by precisely fitting the molecules on their surfaces to the target cell surface membrane. In addition, viruses have vast genetic variation, wide variety in means of transmission, efficient processes of replication within host cells, and the ability to persist in the host seemingly impervious to the immune system response [17]. 


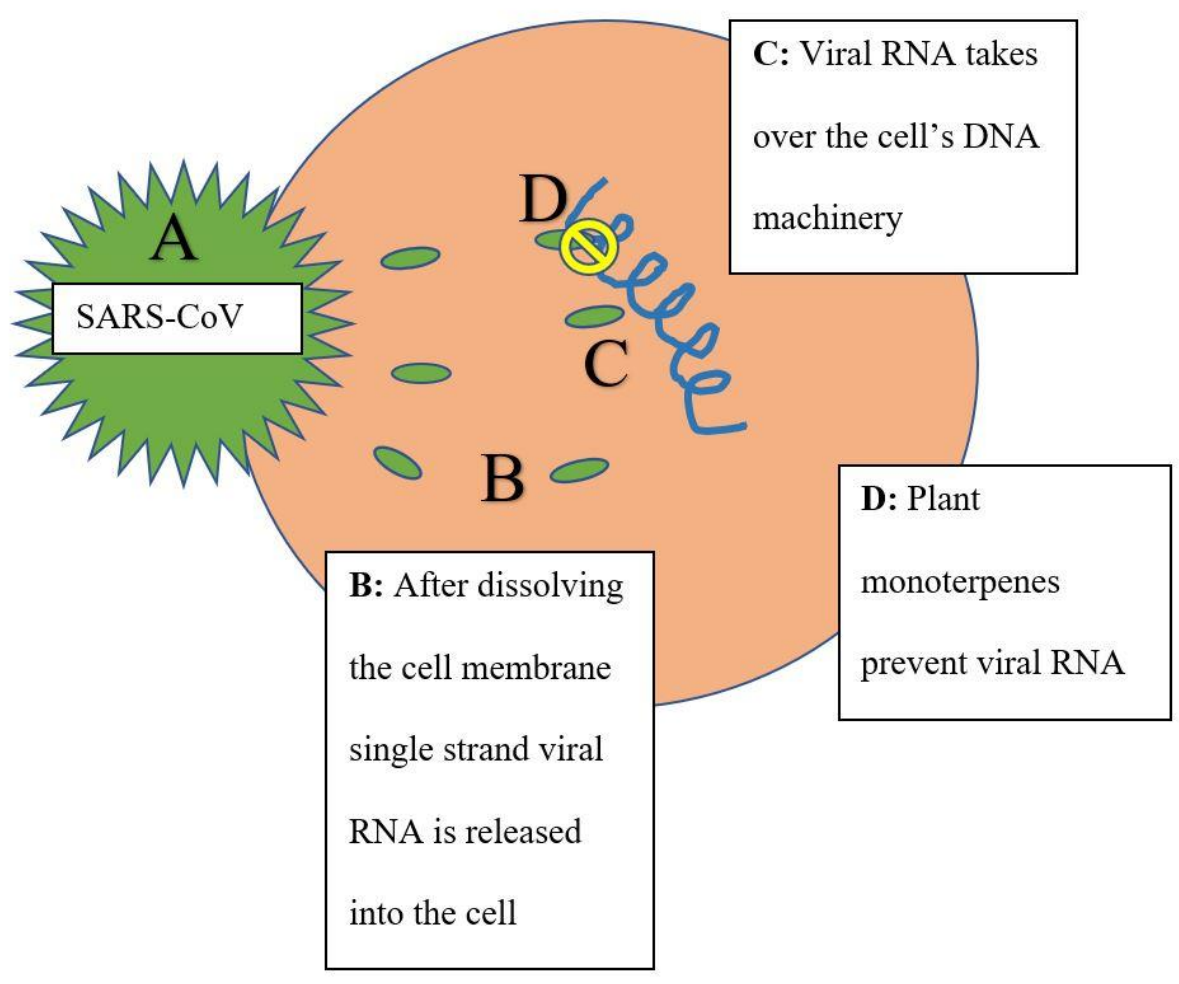

Figure 1 Replication of SARS-CoV virus.

\section{Specific Essential Oils with Potential SARS-CoV Antiviral Properties}

Specific EOs that have been shown to have antiviral properties include Cinnamomum zeylanicum, Daucus carota, Eucalyptus globulus, Syzygium aromaticum and Origanum vulgare [23]. One should note that most of studies highlighted reported synergetic effects of EO's, rather than individual impact. Essential oils are extremely concentrated, complex mixtures of phytochemicals that have as their main constituents' terpenes, terpenoids, and phenylpropanoids that are responsible for their biologic properties. Herbals and essential oils (EOs) have been used in medicine for centuries because of biological properties such as larvicidal, antimicrobial, analgesic and anti-inflammatory, antioxidant, fungicide, and antitumor activities. Of the 250-300 commercially available EOs, many exhibit antimicrobial properties with about a dozen possessing high antimicrobial potential EOs may represent a viable alternative to synthetic and/or pharmaceutical antiviral compounds due to the fact that organisms cannot develop resistance against the $100 \mathrm{~s}$ of phytochemicals fast enough for resistant strains to form. Many of the hundreds of constituent ingredients in EOs alone, or in combination, have been shown to be effective antivirals and antimicrobials [24]. Won-Young Bae and Hyeong-Yeop Kim, et al (2019) evaluated specific known antiviral EOs to determine if they may be 
useful against SARS-CoV. The oils chosen included extracts of three medicinal herbs (Brassica juncea, Forsythia suspensa, and Inula britannica) were prepared using ethanol or water. The total phenolic, flavonoid, and saponin content, condensed tannin content, and reducing sugar content of the herb extracts were determined via phytochemical screening. Of the three medicinal herbs studied, $F$. suspensa showed the highest concentration of phenolic compounds, flavonoids, and saponins; however, the extract of $B$. juncea showed an impressive $3 \log$ TCID50/25 $\mu \mathrm{L}$ virus titration reduction and the water extract showed a selectivity index of 13.668 against infected influenza H1N1 virus with no evidence of hepatotoxicity or hemolysis. Extract of I. britannica showed the most effective virus titration decrease; however, its hemolytic and hepatotoxicity values were the most significant of the three EOs. The authors concluded that of the three EOs tested, $B$. juncea may show antiviral effects against H1N1 in vivo with fewer potential side effects compared to other antiviral agents [25].

Table 1 Proposed mechanism of action of herbal antivirals.

\section{Proposed Mechanism of Action of Essential Oils}

1. The 100 's to 1000 's of phytochemicals act synergistically

2. Chemotoxicity of phytochemicals damages pathogenic cell membranes

3. Lipophilic properties allow EOs to easily pass through the viral membrane

4. Once inside the membrane, EO chemotoxins destroy polysaccharide, fatty acid and phospholipid layers

5. Nanoparticles render the EO impervious to viral defense systems due to chemical stability, sustained release and enhanced bioavailability

6. The hydrophobicity of EOs enables them to partition themselves in cell membrane lipids disturbing viral cell structure and making viral membranes more permeable

7. The permeability results in the death of the virus

8. Signaling cascades are disrupted inhibiting viral replication

9. The end results is the death of the virus.

Some currently available compounds that have been identified at the 17th International Conference on Antiviral Research proceedings by Vijgen L, Keyaerts E, Van Damme E et al., (2004) as SARS-CoV replication inhibitors exhibiting diverse mechanisms of action are the mannose-specific plant lectins derived from Galanthus nivalis (common snowdrop), Hippeastrum hybrids (Amaryllis) and Allium porrum (leek). This group of plant lectins were shown to have inhibitory effects on SARSCoV replication, with selectivity indexes of up to 100 , and EC50 values as low as $1 \mu \mathrm{g} / \mathrm{ml}$ [26]. Licorice root (Glycyrrhiza radix) is a known anti-inflammatory substance in Chinese medicine has long been known as an antiviral substance active in vitro against both RNA and DNA viruses, including HIV. Unfortunately, even though Glycyrrhiza has been shown to inhibit the replication of SARS-CoV, the concentration which is required to be effective is so high that it would be difficult to achieve it in vivo [27]. In a study conducted by Lin and Tsai, et.al (2005) evaluating Isatis indigotica (woad or glastum), is a flowering plant in the family Brassicaceae. I. indigotica root extract was tested for anti-SARS-CoV coronavirus main proteinase (3CLpro) effects using cell-free and cell-based cleavage assays. Of the 12 
compounds in I. indigotica, only two phenolic compounds, aloe emodin and hesperetin dosedependently inhibited cleavage activity of 3CLpro in a cell-based assay [28]. A series of research studies by Li, Chen and Zhang, et al (2005) on the antimicrobial effects of four herbal extracts showed moderate to potent antiviral activities against SARS-CoV at a $50 \%$ effective concentration. Of four herbal extracts evaluated, Lycoris radiata (licorice root) was most potent with identification of a single substance, lycorine, as a potential anti-SARS-CoV component suggesting that the compounds containing lycorine are potential candidates for development of new anti-SARS-CoV treatments (Table 1) [29].

Essential oils from Eucalyptus globulus, M. alternifolia (tea tree) and Thymus vulgaris (thyme) have been examined for potential antiviral activity, specifically by their major monoterpene compounds alpha-terpinene, carvacrol, gamma-terpinene, alpha-pinene, p-cymene, terpinen-4-ol, alpha-terpineol, thymol, citral and 1,8-cineole [19]. M. alternifolia EO has been shown to be effective against various influenza viruses, HSV-1 and SARS-CoV viruses by inhibiting the proliferation of the enveloped virus inside cells, according to research conducted by Lelesius, R. \& Karpovaite, A., et al (2019). Ethanol extracted plant material studied in vitro that interfered with SARS-CoV infections included three very effective EOs including peppermint (Mentha piperita), thyme ( $T$. vulgaris), and showy tick-trefoil (Desmodium canadense) [30]. The antimicrobial activity of T. vulgaris is probably the most wellstudied of the EOs, according to Salehi \& Abu-Darwish, et al (2019) [31]. The main biochemically active components of thyme EO are thymol (36-55\%) and p-cymene (15-28\%). Thyme EO as a compound is characterized by strong bactericidal, antifungal, antiviral and anti-parasitic properties, with relatively low toxicity to humans and animals. When thyme as an EO is used as an antimicrobial, recommended composition of compounds should be in the following ranges: $\gamma$-terpinene (5-10\%), linalool (4-6.5\%), carvacrol (1-4\%), b-myrcene (1-3\%) and terpin-4-ol (0.2-4.0\%). Thymol also contains monoterpene hydrocarbons, such as $\alpha$-tujen, $\alpha$-pinene, $\alpha$-terpinen, camphor myrcen, and the oxygen derivatives of monoterpenes, 1.8-cineol, linalool, terpin-4-ol, and $\gamma$-terpinol, camphor and borneol. Sesquiterpene hydrocarbons in thyme have been identified as including $\alpha$-humulene, $\alpha$ kopene, kubeben, $\alpha$-gurjunen, germacren $D, \alpha$-and $\gamma$-muurolen, and $\gamma$ - and $\delta$-cadinene. In vitro, thyme EO has antiviral activity active against the DNA virus, herpes simplex-1 (HSV1) with half maximal inhibitory concentration (IC50) values of $11 \mu \mathrm{g} / \mathrm{mL}$, and also displayed $100 \%$ inhibitory activity in the liquid phase at $3.1 \mu \mathrm{L} / \mathrm{mL}$ concentration against the RNA virus influenza A1/Denver/1/57 (H1N1) with 30 minute exposure. The mechanism of action of thymol is mainly attributed to its low polarity, which easily interferes with the lipid bilayer of the viral cell membrane, changing its fluidity and permeability rendering it inactive [14, 32]. Another EO, Eucalyptus globulus that has antimicrobial activity against viruses, bacteria, yeasts, and fungi may be promising as well. E. globulus EO, rich in 1,8-cineole (88\%), was active against HSV-1 in vitro and was able to suppress viral multiplication by $>96 \%$. E globulus acts by directly inactivating free-virus particles and might also interfere with virion envelope structures required for entry into host cells (Elaissi, Rouis and Salem, et al [2012]) [33]. Blends of Cinnamomum zeylanicum, Daucus carota, E. globulus and Rosmarinus officinalis EOs were effective against several antibiotic resistant strains of bacteria, and also against influenza (H1N1) viruses in vitro [3]. Chemical analysis of inhibitory activity by EO's against SARS-CoV and HSV-1 replication in vitro was conducted by Loizzo, Saab, et al., (2008) by visually scoring of the virus-induced cytopathogenic effect post- 
infection. Laurus nobilis (Bay Laurel) EO exerted an interesting activity against SARS-CoV with an IC (50) value of $120 \mathrm{microg} / \mathrm{ml}$ and a selectivity index (SI) of 4.16. L. nobilis EO was characterized by the presence of beta-ocimene, 1,8-cineole, alpha-pinene, and beta-pinene as main constituents. Additionally, Juniperus oxycedrus (Cade) EO, in which alpha-pinene and beta-myrcene were major constituents, revealed antiviral activity against HSV-1 with an IC (50) value of $200 \mathrm{microg} / \mathrm{ml}$ and a SI of 5 [34].

Of the very few studies that look at the use of phytochemicals from plants specifically against the SARS-CoV RNA virus, a large in vitro study by Wen \& Kuo, et al., (2007) of 221 phytocompounds against SARS-CoV that included ten diterpenoids, two sesquiterpenoids, two triterpenoids, five lignoids, curcumin, and reference controls of niclosamide and valinomycin (known potent inhibitors) at concentrations between 3.3 and 10 microM. Of the compounds studied, betulinic acid and savinin were competitive inhibitors of SARS-CoV $3 \mathrm{CL}$ protease with Ki values $=8.2+/-0.7$ and $9.1+/-2.4$ microM, respectively. Additional findings suggest that specific abietane-type diterpenoids and lignoids exhibited the strongest anti-SARS-CoV effects [35]. A study by Loizzo and Saab, et al (2008) evaluating the potential for a single EO, sage (Salvia officinalis) against SARS-CoV replication demonstrated that both the raw plant material as well as the extracts and EO obtained from $S$. officinalis was active against SARS-CoV viruses obtained from the sputum of a patient hospitalized with a diagnosis of SARS in Frankfurt University Hospital. It is worth noting that the overriding clinical feature of SARS is the rapidity with which many patients develop symptoms of acute respiratory distress syndrome (ARDS) . Sage EO was effective against development of ARDS at IC50 $=870 \mathrm{mg} / \mathrm{mL}$ [34]. In similar studies of the H1N1 (swine flu) RNA virus, sage EO was effective in reducing viral infectivity by $>96 \%$. According to an extensive review by De Clercq (2006), when sage EO was studied against HSV, the isolated monoterpenes alone inhibited HSV by about $>80 \%$ [27].

Another potential plant source of antiviral activity against SARS-CoV is Angelica keiskei, commonly known under the Japanese name of ashitaba which means "tomorrow's leaf". A. keiskei is a species of flowering plant in the carrot family. Park, Ko, and Kim (2016) evaluated nine alkylated chalcones and four coumarins isolates from A. keiskei were evaluated for their activity against SARS-CoV. The authors concluded that of the isolated alkylated chalcones, only chalcone-6, which contains a perhydroxyl group, exhibited the most potent $3 \mathrm{CL}$ (pro) and PL (pro) inhibitory activity with IC50 values of 11.4 and $1.2 \mu \mathrm{M}$ [36]. Another herbal of interest against SARS-CoV that is used in Traditional Chinese medicine for respiratory viruses is Strobilanthes cusia (Nees). Methanol extracts of $S$. cusia leaf were found to contain chemical components such as B-sitosterol, indirubin, tryptanthrin, betulin, indigodole $A$, and indigodole $B$ that have diverse biological activities including antimicrobial activity. An extract of $S$. cusia leaf was shown to inhibit human coronavirus NL63 (HCoV-NL63) viral infection in a concentration-dependent manner by Tsai, Lee and Yen, et al (2019). Among the six components identified in the methanol extract of $S$. cusia leaf were tryptanthrin and indigodole $B$ (5aRethyltryptanthrin); of which tryptanthrin exhibited the most potent antiviral activity in reducing the CPE and progeny virus production giving it the strongest antiviral potential against HCoV-NL63 infection [37]. In vitro examination by Weng and Lin (2019) of Sambucus FormosanaNakai, a species of elderberry which is a traditional medicinal herb with anti-inflammatory and antiviral activity for potential use against HCoV-NL63 found that S. FormosanaNakai interrupted the replication 
capabilities of the virus. The mechanism of action was thought to be the constituent phytochemicals in S. FormosanaNakai (caffeic acid, chlorogenic acid and gallic acid) of which caffeic acid most significantly inhibited replication of HCoV-NL63 in a cell-type independent manner, and specifically blocked virus attachment (IC50 = 8.1 $\mu \mathrm{M})$ (Figure 1) [38].

A key enzyme in the processing of viral polyproteins thereby contributing significantly to the biogenesis of viral replication in SARS-CoV is the papain-like protease (PLpro) complex. Coronavirus proteases, PLpro and $3 \mathrm{C}$-like protease (3CLpro), are attractive antiviral chemical targets because those proteases are essential for coronavirus replication. The primary function of PLpro and 3CLpro are to process the viral polyprotein in a coordinated manner; however, PLpro has the additional function of stripping ubiquitin and interferon-stimulated gene-15 (ISG15) from host-cell proteins to aid coronaviruses in their evasion of the host innate immune responses [2]. Studies have shown that PLpro requires a zinc-binding domain and a catalytic triad (Cys1651-His1812-Asp1826) instead of a catalytic dyad for protease activity [39]. Asp1826 and the four cysteine residues involved in zinc binding are essential for SARS-CoV PLpro activity. Molecular modeling and analysis of activity of SARSCoV PLpro by Barretto and Jukneliene et al (2015) determined that this catalytic core may also have deubiquitinating activity. Further examination of the activity of SARS CoV-PLpro found that it hydrolyzed both diubiquitin and ubiquitin-7-amino-4-methylcoumarin (AMC) substrates. Hydrolysis of ubiquitin-AMC is thought to be approximately 180 -fold more efficient than hydrolysis of a peptide substrate that mimics the PLpro replicase recognition sequence [39]. Additionally, it was discovered that PLpro recognizes the consensus cleavage sequence $L X G G$, which is also the consensus sequence recognized by cellular deubiquitinating enzymes. Barretto and Jukneliene concluded that the similarity in the substrate recognition sites should be an important consideration during the development of SARS-CoV PLpro inhibitors [39].

In vitro research was conducted by Kim and Seo, et al (2014) to examine the potential of a plant extract from Psoralea corylifolia (common name is babchi in Asia) to interrupt PLpro deubiquitinating enzymes. P. corylifolia has been used traditionally for skin-related conditions such as psoriasis, vitiligo and is used because its chief constituent psoralen is a photoactive furocoumarin that binds to DNA when exposed to UV light to form photoproducts with pyrimidine base. A review of the traditional uses and constituent ingredients in $P$. corylifolia found a wide range of phytochemical constituents that have been isolated from the plant possessing important bioactive functions that are antimicrobial, antibacterial, antiviral, antifungal anti-inflammatory, antitumor, hepatoprotective, antipsoriasis, anthelmintic, antidiabetic and immunomodulation. The major components in extracts from P. corylifolia, psoralen and isopsoralen, have been identified as the phytochemical components with the highest antitumor, antibacterial, and antiviral properties [40]. Research evaluating the phytochemicals in P. corylifolia uncovered the presence of fourteen compounds including aromatic, sesquiterpenes, furocoumarins, sterols, fatty acid and their methyl esters. $P$. corylifolia seeds contain unique phytochemicals with considerable antimicrobial activity validating their use in traditional medicine and suggesting their potential as a source of new natural antimicrobial compounds. Bioassay directed purification assays of the active compounds in $P$. corylifolia identified new compounds, corylifolin-1, and bakuchiol-2, which are both potent DNA polymerase inhibitors [41]. Ethanol extracts from the seeds of $P$. corylifolia found that the extract showed high activity against 
the SARS-CoV PLpro with an IC50 of value of $15 \mathrm{microg} / \mathrm{ml}$ [42]. Evaluation of the bioactivity-guided fractionation of the ethanol extract of $P$. corylifolia uncovered six aromatic compounds which were identified as bavachinin, neobavaisoflavone, isobavachalcone, 4'-O-methylbavachalcone, psoralidin and corylifol $A$. All six of the isolated flavonoids inhibited PLpro in a dose-dependent manner with IC50 ranging between 4.2 and $38.4 \mu \mathrm{M}$ [42].

\section{Summary}

SARS-CoV represents a novel group of RNA viruses that rapidly develop into deadly illness within a matter of days to weeks. There are no known treatments for prevention or eradication of SARS-CoV to date. Antiviral medications that have been used to treat or control other viral infections have been ineffective and therefore, it is imperative to consider alternatives to current antiviral treatment options for this disease. Research targeting PLpro with antiviral drugs may provide an answer to inhibiting viral replication as well as inhibiting the dysregulation of signaling cascades in infected cells leading to cell death in surrounding, uninfected cells. P. corylifolia seed extracts and certain EOs have been used successfully as antimicrobials for centuries and have been shown to be effective against various types of pathogenic viruses including SARS-CoV in vitro. Of the EO choices available, those highest in monoterpenes seem the most likely to be effective that should be studied against SARSCoV include Salvia officinalis (sage), Psoralea corylifolia seed extracts, Melaleuca alternifolia, Thymus vulgaris (thyme) and Brassica juncea EOs.

\section{Acknowledgments}

Thank you to Dr. Denise Wood and Dr. Chris Nutting at Huntington University of Health Sciences for your ongoing and tireless support of my work.

\section{Author Contributions}

$100 \%$ of the contribution of this work was completed by the author, Sharon Freeman Clevenger.

\section{Competing Interests}

The author has declared that no competing interests exist.

\section{References}

1. Wu D, Wu T, Liu Q, Yang Z. The SARS-CoV-2 outbreak: What we know. Int J Infect Dis. 2020; 94: 4448.

2. Baez-Santos YM, St John SE, Mesecar AD. The SARS-coronavirus papain-like protease: Structure, function and inhibition by designed antiviral compounds. Antiviral Res. 2015; 115: 21-38.

3. Brochot A, Guilbot A, Haddioui L, Roques C. Antibacterial, antifungal, and antiviral effects of three essential oil blends. Microbiologyopen. 2017; 6: e00459.

4. Chouhan S, Sharma K, Guleria S. Antimicrobial activity of some essential oils-present status and 
future perspectives. Medicines (Basel). 2017; 4: 58.

5. Infectious diseases [Internet]. Washington D.C.: Centers for Disease Control; 2017 [cited 202004 24]. Available from: https://www.cdc.gov/nchs/fastats/infectious-disease.htm.

6. Cheng VCC, Lau SKP, Woo PCY, Yuen KY. Severe acute respiratory syndrome coronavirus as an agent of emerging and reemerging infection. Clin Microbiol Rev. 2007; 20: 660-94.

7. Woo PC, Lau SK, Yuen KY. Infectious diseases emerging from Chinese wet-markets: Zoonotic origins of severe respiratory viral infections. Curr Opin Infect Dis. 2006; 19: 401-407.

8. Alberts B. Pathogens and infection. Molecular biology of the cell. 6th ed. NY: W. W. Norton \& Company; 2015. p. 1263-1296.

9. McAdam AJ, Milner DA, Sharpe AH. Infectious diseases. Robbins and cotran pathologic basis of disease. 9th ed. Philadelphia, PA: Elsevier Saunders; 2015. p. 341-402.

10. Overview of viral infections [Internet]. NY: Merck Manual; 2018 [cited 202004 24]. Available from: https://www.merckmanuals.com/home/infections/overview-of-viral-infections/overviewof-viral-infections.

11. Weiss SR, Leibowitz JL. Chapter 4 - Coronavirus pathogenesis. Advances in virus research. 81th ed. MA: Academic Press; 2011. p. 85-164.

12. WHO. Coronavirus disease 2019 [Internet]. Geneva: World Health Organization; 2020 [cited 2020 04 24]. Available from: https://www.who.int/emergencies/diseases/novel-coronavirus-2019.

13. Siu KL, Yuen KS, Castano-Rodriguez C, Ye ZW, Yeung ML, Fung SY, et al. Severe acute respiratory syndrome coronavirus ORF3a protein activates the NLRP3 inflammasome by promoting TRAF3dependent ubiquitination of ASC. FASEB J. 2019; 33: 8865-8877.

14. Biological sciences curriculum study: Understanding emerging and re-emerging infectious diseases [Internet]. Bethesda: National Institutes of Health; 2007 [cited 202004 24]. Available from: https://www.ncbi.nlm.nih.gov/books/NBK20370/.

15. Schnaubelt $\mathrm{K}$. The healing intelligence of essential oils: The science of advanced aromatherapy. Rochester, VT: Healing Arts Press; 2011. 234 p.

16. Lin $\mathrm{MH}$, Moses $\mathrm{DC}$, Hsieh $\mathrm{CH}$, Cheng SC, Chen $\mathrm{YH}$, Sun $\mathrm{CY}$, et al. Disulfiram can inhibit MERS and SARS coronavirus papain-like proteases via different modes. Antiviral Res. 2018; 150: 155-63.

17. Jassim SA, Naji MA. Novel antiviral agents: A medicinal plant perspective. J Appl Microbiol. 2003; 95: 412-27.

18. Giraud-Robert AM. The role of aromatherapy in the treatment of viral hepatitis. Int J Aromatherapy. 2005; 15: 183-92.

19. Astani A, Reichling J, Schnitzler P. Comparative study on the antiviral activity of selected monoterpenes derived from essential oils. Phytother Res. 2010; 24: 673-679.

20. Carvacrol, $\mathrm{CID}=10364$ [Internet]. Bethesda: National center for biotechnology information; 2020 [cited 202004 24]. Available from: https://pubchem.ncbi.nlm.nih.gov/compound/Carvacrol.

21. Baudoux D. Antiviral and antimicrobial properties of essential oils [Internet]. Pranarom: Positive health online; 2000 [cited $2020 \quad 04$ 24]. Available from: http://www.positivehealth.com/article/aromatherapy/antiviral-and-antimicrobial-properties-ofessential-oils.

22. Worwood V. The Self-Defense Kit. The complete book of essential oils \& aromatherapy. 25th ed. 
Novato, CA: New World Library; 2016. p. 49-66.

23. Shreaz S, Bhatia R, Khan N, Muralidhar S, Basir SF, Manzoor N, et al. Spice oil cinnamaldehyde exhibits potent anticandidal activity against fluconazole resistant clinical isolates. Fitoterapia. 2011; 82: 1012-1020.

24. Winska K, Maczka W, Lyczko J, Grabarczyk M, Czubaszek A, Szumny A. Essential oils as antimicrobial agents-myth or real alternative? Molecules. 2019; 24: 2130.

25. Bae WY, Kim HY, Choi KS, Chang KH, Hong YH, Eun J, et al. Investigation of brassica juncea, forsythia suspensa, and inula britannica: Phytochemical properties, antiviral effects, and safety. BMC Complement Altern Med. 2019; 19: 253.

26. Vijgen L, Keyaerts E, Van Damme E, Peumans W, De Clercq E, Balzarini J, et al. Antiviral effect of plant compounds of the Alliaceae family against the SARS coronavirus. Antiviral Res. 2004; 62: A76

27. De Clercq E. Potential antivirals and antiviral strategies against SARS coronavirus infections. Expert Rev Anti Infect Ther. 2006; 4: 291-302.

28. Lin CW, Tsai FJ, Tsai CH, Lai CC, Wan L, Ho TY, et al. Anti-SARS coronavirus 3C-like protease effects of Isatis indigotica root and plant-derived phenolic compounds. Antiviral Res. 2005; 68: 36-42.

29. Li SY, Chen C, Zhang HQ, Guo HY, Wang H, Wang L, et al. Identification of natural compounds with antiviral activities against SARS-associated coronavirus. Antiviral Res. 2005; 67: 18-23.

30. Lelesius R, Karpovaite A, Mickiene R, Drevinskas T, Tiso N, Ragazinskiene O, et al. In vitro antiviral activity of fifteen plant extracts against avian infectious bronchitis virus. BMC Vet Res. 2019; 15: 178.

31. Salehi B, Abu-Darwish MS, Tarawneh AH, Cabral C, Gadetskaya AV, Salgueiro L, et al. Thymus spp. plants - food applications and phytopharmacy properties. Trends Food Sci Technol. 2019; 85: 287306.

32. Vergis J, Gokulakrishnan P, Agarwal RK, Kumar A. Essential oils as natural food antimicrobial agents: A review. Crit Rev Food Sci Nutr. 2015; 55: 1320-1323.

33. Elaissi A, Rouis Z, Salem NAB, Mabrouk S, ben Salem Y, Salah KBH, et al. Chemical composition of 8 eucalyptus species' essential oils and the evaluation of their antibacterial, antifungal and antiviral activities. BMC Complement Altern Med. 2012; 12: 81.

34. Loizzo MR, Saab AM, Tundis R, Statti GA, Menichini F, Lampronti I, et al. Phytochemical analysis and in vitro antiviral activities of the essential oils of seven lebanon species. Chem Biodivers. 2008; 5: 461-70.

35. Wen CC, Kuo YH, Jan JT, Liang PH, Wang SY, Liu HG, et al. Specific plant terpenoids and lignoids possess potent antiviral activities against severe acute respiratory syndrome coronavirus. J Med Chem. 2007; 50: 4087-4095.

36. Park JY, Ko JA, Kim DW, Kim YM, Kwon HJ, Jeong HJ, et al. Chalcones isolated from angelica keiskei inhibit cysteine proteases of SARS-CoV. J Enzyme Inhib Med Chem. 2016; 31: 23-30.

37. Tsai YC, Lee CL, Yen HR, Chang YS, Lin YP, Huang SH, et al. Antiviral action of tryptanthrin isolated from strobilanthes cusia leaf against human coronavirus NL63. Biomolecules. 2020; 10: 366.

38. Weng JR, Lin CS, Lai HC, Lin YP, Wang CY, Tsai YC, et al. Antiviral activity of sambucus formosanaNakai ethanol extract and related phenolic acid constituents against human 
coronavirus NL63. Virus Res. 2019; 273: 197767.

39. Barretto N, Jukneliene D, Ratia K, Chen Z, Mesecar AD, Baker SC. The papain-like protease of severe acute respiratory syndrome coronavirus has deubiquitinating activity. J Virol. 2005; 79 : 15189-15198.

40. Liu R, Li A, Sun A, Kong L. Preparative isolation and purification of psoralen and isopsoralen from Psoralea corylifolia by high-speed counter-current chromatography. J Chromatogr A. 2004; 1057 : 225-228.

41. Nabi NG, Shrivastava M. Endangered medicinal plant psoralea corylifolia: Traditional, phytochemical, therapeutic properties and micropropagation. UK JPB. 2017; 5: 40-6.

42. Kim DW, Seo KH, Curtis-Long MJ, Oh KY, Oh JW, Cho JK, et al. Phenolic phytochemical displaying SARS-CoV papain-like protease inhibition from the seeds of psoralea corylifolia. J Enzyme Inhib Med Chem. 2014; 29: 59-63.

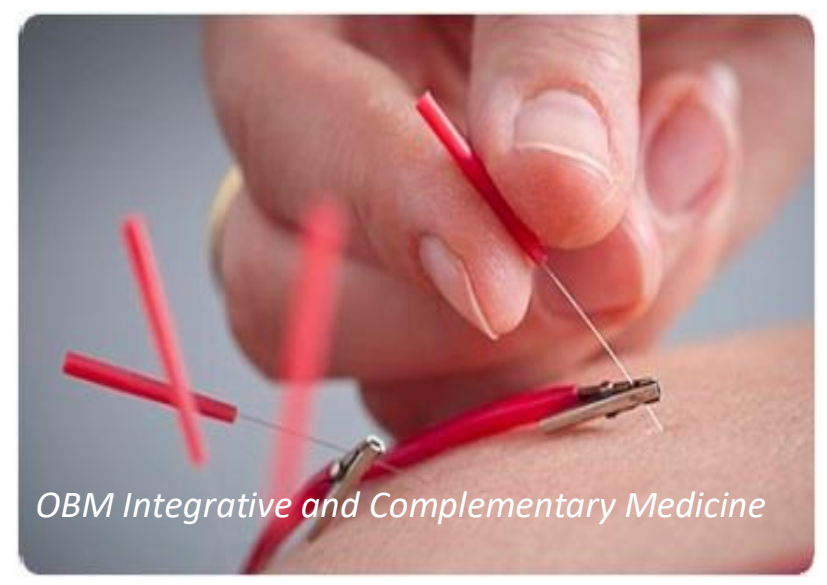

Enjoy OBM Integrative and Complementary Medicine by:

1. Submitting a manuscript

2. Joining in volunteer reviewer bank

3. Joining Editorial Board

4. Guest editing a special issue

For more details, please visit: http://www.lidsen.com/journals/icm 